\title{
A Blind Equalization Algorithm Based on Global Artificial Fish Swarm and Genetic Optimization DNA Encoding Sequences
}

\author{
Hui Wang ${ }^{\text {a }}$, Yecai Guo ${ }^{\text {b }}$ \\ College of Electronic \& Information Engineering, Nanjing University of Information Science \& \\ Technology, Nanjing, 210044, China \\ aw_hui75@163.com, bguo-yecai@163.com
}

Keywords: Stochastic conjugate gradient multi-modulus algorithm (SMMA); Global artificial fish swarm algorithm (GAFSA); DNA genetic algorithm (DNA-GA)

\begin{abstract}
A stochastic conjugate gradient multi-modulus algorithm based on global artificial fish swarm and genetic optimization DNA encoding sequences(GAFS-GDNA- SMMA) is proposed to solve the high computational loads, low convergence rate, and large mean square error(MSE) of the multi-modulus blind equalization algorithm(MMA). In this proposed algorithm, the optimal DNA coding sequence can be found and used as the initial optimal weight vector of the stochastic conjugate gradient MMA(SMMA) after decoding the optimization DNA sequences. The simulation results show that the proposed algorithm has the faster convergence speed and smaller mean square error comparison with MMA, SMMA, GA-DNA-SMMA(Genetic algorithm and DNA optimization based SMMA)
\end{abstract}

\section{Introduction}

In order to overcome the low convergence rate, high computational loads, and large mean square error(MSE) of the multi-modulus blind equalization algorithm(MMA), it is necessary to propose a novel multi-modulus blind equalization algorithm. Therefore, the conjugate gradient method with quadratic convergence rate is introduced into the MMA to quickly find the optimal solution. The stochastic conjugate gradient MMA(SMMA) can reduce the amount of calculation greatly and still has faster convergence speed and small mean square error comparison with the multi-modulus blind equalization algorithm(MMA).

The DNA genetic algorithm(DNA-GA) has the flexible encoding and higher encoding accuracy. Besides, it has the ability of global search. The global artificial fish swarm algorithm (GAFSA) is a new and highly efficient intelligence algorithm with the global searching ability and can overcome the defects of the low precision, slow convergence, and high complexity of the basic artificial fish swarm algorithm. So, the DNA-GA fusing into GAFSA can improve the ability of global search and find the best DNA sequence quickly.

In this paper, the stochastic conjugate gradient multi-modulus blind equalization algorithm based on global artificial fish swarm and genetic optimization DNA encoding sequence(GAFS- GDNASMMA) is proposed via fusing the advantages of the MMA, the DNA-GA, and the GAFSA.

\section{DNA Genetic Algorithm Based on GASFA}

The global artificial fish swarm algorithm(GAFSA) is an efficient optimal algorithm with preying, swarming, and following behavior of fish swarm. In a watering place, the fish can find the place which has a lot of food through the preying, swarming and following behavior. The artificial fish swarm can achieve the purpose of optimization according to the feature of the fish swarm.

The DNA genetic algorithm(DNA-GA) has the advantages of traditional genetic algorithm. Besides, it is a good optimization method with the flexible encoding and high precision.

In crossover operator, two individuals in the population are selected as the parent bodies. One sequence, which belongs to one parent body, is randomly selected. These two sequences have the same length and are exchanged to come into being two new individuals.

In mutation operator, each base of each individual in the group can mutate into another base with 
a certain probability to form a new population.

Therefore, the GAFSA is fused with DNA-GA and carried out. DNA-GA with global artificial fish swarm algorithm is proposed to improve the ability of the global search and find the optimal value quickly and accurately.

\section{Novel Multi-Modulus Blind Equalization Algorithm}

The stochastic conjugate gradient multi-modulus algorithm(SMMA) adopts the conjugate gradient method in the updating process of the weight vector. The updating direction of every step in the updating process is composed of the gradient direction of the current iterative point and the searching direction of the previous iterative point. New searching direction including the gradient information of the current and previous points can reflect the current searching direction and make the equalizer coefficient converge to the global optimal point.

The cost function of the SMMA is defined as

$$
J_{\text {SCGMMA }}(n)=E\left\{\frac{\left|y_{R}(n)\right|^{p+2}}{p+2}-\frac{y_{R}^{2}(n) R_{R}^{p}}{2}+\frac{\left|y_{I}(n)\right|^{p+2}}{p+2}-\frac{y_{I}^{2}(n) R_{I}^{p}}{2}\right\}
$$

where $p=2, R_{R}^{p}$ and $R_{I}^{p}$ are the real and imaginary parts of modulus, respectively. $y_{R}(n)$ and $y_{I}(n)$ is the quadrature and in-phase component of the decision output signals.

According to Eq.(1), the equalizer tap coefficient vector of the SMMA is updated by

$$
\begin{aligned}
& \boldsymbol{W}(n+1)=\boldsymbol{W}(n)+\mu \boldsymbol{d}(n) \\
& \boldsymbol{d}(n)=\left\{\begin{array}{c}
\boldsymbol{g}(n), \quad n=1 \\
\boldsymbol{g}(n)+\beta(n-1) \boldsymbol{d}(n-1), n>1
\end{array}\right. \\
& \boldsymbol{g}(n)=\left[y_{R}(n)\left(R_{R}^{p}-\left|y_{R}(n)\right|^{p}\right)+j y_{I}(n)\left(R_{I}^{p}-\left|y_{I}(n)\right|^{p}\right)\right] \boldsymbol{X}^{*}(n)
\end{aligned}
$$

where $\beta(n)=\frac{\|g(n+1)\|^{2}}{\|g(n)\|^{2}} . \mu$ is the step-size.

The hamming distance between two DNA sequences is the sum of the different characters in all the corresponding position to describe that the two DNA sequences aren't similar degree. DNA sequence $X$ and $Z$ are denoted as $X=\mathrm{x} 1 \mathrm{x} 2 \ldots \mathrm{xn}$ and $Z=\mathrm{z} 1 \mathrm{z} 2 \ldots \mathrm{zn}$, where $n$ is the number of the DNA bases in $X$ or $Z$, xm and zl are the any kind of four bases named as Adenine, Guanine, Cytosine, and Thymine. Their hamming distance is written as $H(X, Z)$ and defined as

$$
H(X, Z)=\sum_{i=1}^{n} h\left(x_{i}, z_{i}\right)
$$

where the value of $h\left(x_{i}, z_{i}\right)$ is zero if $x_{i}$ is equal to $z_{i}$, else the value is 1 .

In the design of DNA encoding, the number of different bases in the sequence $X$ and $Y$ increases with the increase of hamming distance. So, the possibility of hybridization becomes small.

There are two fitness functions in this paper. The first one is used to handle constraints with the weighted average method and defined as

$$
Y_{1}\left(s_{i}\right)=w F\left(s_{i}, s_{i}\right)=w \cdot \min _{-n<k<n} H\left(s_{i}, \sigma^{k}\left(s_{i}^{R}\right)\right)
$$

where $s_{i}$ is corresponds to the $i$ th DNA sequence, equal to the ith position vector of artificial fish swarm. $w$ is the weight vector of the constraint $\mathrm{F}\left(\boldsymbol{s}_{i}, \boldsymbol{s}_{i}\right)$, for simplicity, we use that $\mathrm{w}=1$.

The second fitness function is defined as the reciprocal of the cost function of the MMA, i.e.

$$
Y_{2}\left(v_{i}\right)=\frac{1}{J_{S M M A}\left(f_{i}\right)}
$$

where $J_{\text {SMMA }}\left(f_{i}\right)$ is the cost function of the SMMA.

Step1: Initialization of DNA sequence. The initial population of the DNA sequence $s=$ $\left[\boldsymbol{s}_{1}, \boldsymbol{s}_{2}, \ldots, \boldsymbol{s}_{N}\right]$, where $\boldsymbol{s}_{m}$ is the $m$ th DNA sequence and $N$ is the number of the DNA sequence.

Step2: DNA coding and the calculation of the second fitness function. The second fitness 
function of each individual is calculated according to the Eq.(7). The maximum fitness function and its corresponding to the DNA coding value of DNA sequence are recorded in the bulletin board.

Step3: The DNA genetic algorithm(DNA-GA). According to the Eq.(6), the crossover operator and mutation operator are carried out for DNA sequences. Two sequences are selected in DNA sequences as the parent bodies. The translocation crossover operator can be implemented if the random number within 0 to 1 is less than the crossover probability. A random number within 0 to 1 is selected again and compared with the mutation probability. If the random number is small, the mutation operator is carried out. The new population of the DNA sequences is obtained through the two operators.

Step4: The calculation of the first fitness function. The first fitness function of each DNA sequence is calculated according to the Eq.(6). The population of the DNA sequences is sorted according to the first fitness function values from small to large and divided into superior group and inferior group.

Step5: The global artificial fish swarm algorithm(GAFSA). The position vectors of the global artificial fish swarm are obtained by DNA coding of the DNA sequences in the inferior group. Each artificial fish in the fish swarm carries out following behavior. If the following behavior doesn't occur, all fishes carry out swarming behavior. If swarming behavior does also not succeed, all fishes carry out preying behavior. According to DNA encoding, the position vectors of artificial fish swarm change into the DNA sequences. The new population of the DNA sequences is obtained.

Step6: DNA coding and the update of the bulletin board. After the once iteration, the second fitness functions of the population which are obtained by the DNA coding are calculated. The maximum one of them is selected and compared with the previous maximum value of the recorded in the bulletin board. If the maximum one of them is greater than the previous recorded in the bulletin board, the bulletin board is updated by the current maximum value and its corresponding to the DNA coding value.

Step7: Determination of the termination condition. Judge whether the iteration is equal to the maximum value. If the condition doesn't hold, the iteration process goes back to step 3, otherwise, the record in second bulletin board is outputted.

Step8: the record in the bulletin board is used as the initial optimization weight vector to update the weight vector of the SMMA according to Eq.(2).

\section{Test results}

In the simulations, the transmitted signals were 16QAM signals, the underwater acoustic channel

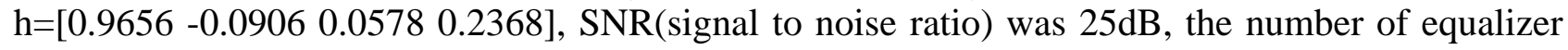
weight coefficients $\mathrm{L}=11$, the step-size $\mu=0.000005$. The number of AFS $N=10$, crowding factor $\delta=0.5$. Figure 1 is simulated results. Figure $1(\mathrm{a})$ is the curves of the MSE, (b) is the constellations of the 16QAM, (c) is input constellations of equalizer, (d) is the output constellations of the MMA, (e) is the output constellations of the SMMA, (f) is the output constellations of the GDNA-SMMA. Figure1(g) is the output constellations of the GAFS-GDNA-SMMA.

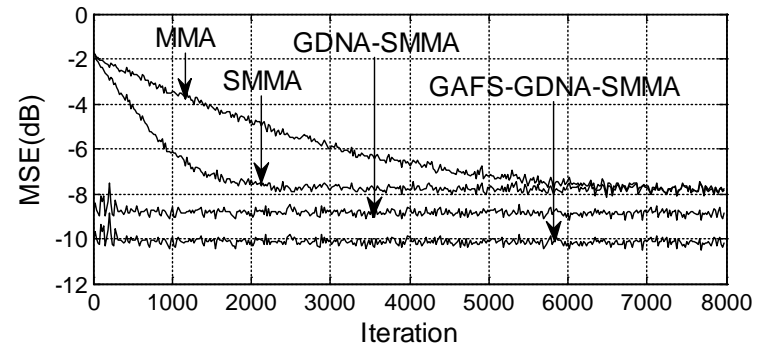

(a)

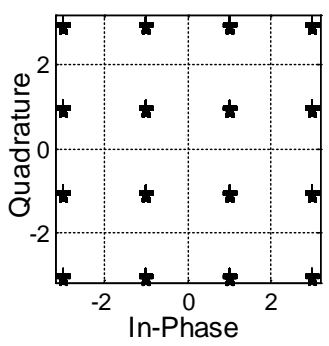

(b)

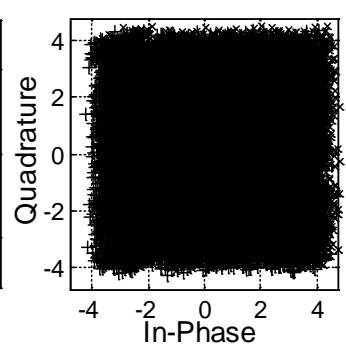

(c) 


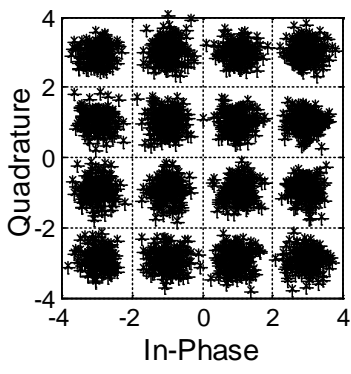

(d)

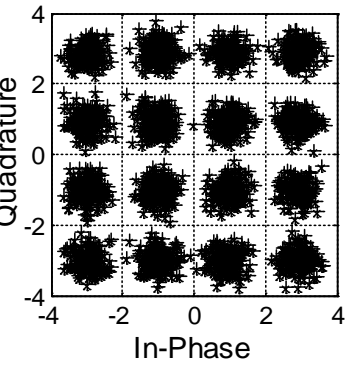

(e)

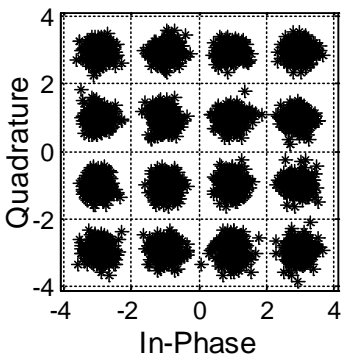

(f)

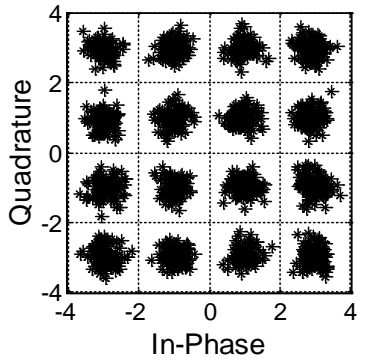

(g)

Figure 1: Simulated results

From Figure 1, we can know that the GAFS-GDNA-SMMA outperforms the GDNA-SMMA, SMMA, and MMA in improving the convergence speed and reducing the MSE. The MSE of the GAFS-GDNA-SMMA is smallest and its convergence speed is fastest. Moreover, the output constellations of the GAFS-GDNA-SMMA are more compact and clearer than other MMAs.

\section{Conclusion}

DNA encoding sequences optimized by fusing DNA-GA with GAFS are applied to the stochastic conjugate gradient multi-modulus algorithm to optimize further the initial weight vector of the SMMA, the SMMA based on DNA encoding sequences optimized by global artificial fish swarm and genetic algorithm is proposed. The simulation results show that the MSE of the GAFS-GDNA-SMMA has fastest convergence rate and lowest MSE, and more compact and clearer output constellations comparison with the GDNA-SMMA, the SMMA, and the MMA.

\section{Acknowledgment}

This work is supported by the major project of University Natural Science of Jiangsu Province (No.13KJA510001), Jiangsu Scientific Research Achievements in Industrialization Project (JHB 2012-9), and A Project Funded by the Priority Academic Program Development of Jiangsu Higher Education Institutions(PAPD II), as well as Innovation Project of Postgraduate Training in Jiangsu Province(No. SJZZ_0110, SJZZ_0111, and KYLX_0853).

\section{References}

[1] Xiao Chen, NingWang, A DNA based genetic algorithm for parameter estimation in the hydrogenation reaction. Chemical Engineering Journal(2009). P. 527-535.

[2] Hai Ma, Yanjiang Wang, An artificial fish swarm algorithm based on chaos Search. Fifth International Conference on Natural Computation. Vol: 4(2009), p.118-121.

[3] WEN Siyuan, LIU Feng. A Computationally Efficient Multi-Modulus Blind Equalization Algorithm. IEEE.Information Management and Engineering(2010). p. 685- 687.

[4] Guangzhao Cui. The Optimization of DNA Encoding Sequences Based on Improved AFS Algorithms. IEEE(2007). p. 1141-1144.

[5] Rezaul Hasan, S.M, A novel mixed-signal integrated circuit model for DNA-protein regulatory genetic circuits and genetic state machines. IEEE Transaction on Circuits and Systems(2010), p.1185-1196. 